\title{
Chiral Spin Texture in the Charge-Density-Wave Phase of the Correlated Metallic Pb/Si(111) Monolayer
}

\author{
C. Tresca, ${ }^{1,2}$ C. Brun, ${ }^{2,}$ T. Bilgeri, ${ }^{2}$ G. Menard ${ }^{2}$ V. Cherkez,${ }^{2}$ R. Federicci, ${ }^{2}$ D. Longo, ${ }^{2}$ F. Debontridder, ${ }^{2}$ \\ M. D'angelo, ${ }^{2}$ D. Roditchev, ${ }^{2,3}$ G. Profeta, ${ }^{1}$ M. Calandra, ${ }^{2, \dagger}$ and T. Cren ${ }^{2}$ \\ ${ }^{1}$ Department of Physical and Chemical Sciences and SPIN-CNR, University of L'Aquila, Via Vetoio 10, I-67100 L'Aquila, Italy \\ ${ }^{2}$ Sorbonne Université, CNRS, Institut des Nanosciences de Paris, UMR7588, F-75252, Paris, France \\ ${ }^{3}$ Laboratoire de physique et d'étude des matériaux, LPEM-UMR8213/CNRS-ESPCI ParisTech-UPMC, \\ 10 rue Vauquelin, F-75005 Paris, France
}

(Received 2 October 2017; revised manuscript received 23 March 2018; published 11 May 2018)

\begin{abstract}
We investigate the $1 / 3$ monolayer $\alpha-\mathrm{Pb} / \mathrm{Si}(111)$ surface by scanning tunneling spectroscopy (STS) and fully relativistic first-principles calculations. We study both the high-temperature $\sqrt{3} \times \sqrt{3}$ and low-temperature $3 \times 3$ reconstructions and show that, in both phases, the spin-orbit interaction leads to an energy splitting as large as $25 \%$ of the valence-band bandwidth. Relativistic effects, electronic correlations, and $\mathrm{Pb}$-substrate interaction cooperate to stabilize a correlated low-temperature paramagnetic phase with well-developed lower and upper Hubbard bands coexisting with $3 \times 3$ periodicity. By comparing the Fourier transform of STS conductance maps at the Fermi level with calculated quasiparticle interference from nonmagnetic impurities, we demonstrate the occurrence of two large hexagonal Fermi sheets with in-plane spin polarizations and opposite helicities.
\end{abstract}

DOI: 10.1103/PhysRevLett.120.196402

Two-dimensional materials with spin-polarized surface states are promising candidates for spin-charge current conversion via the Edelstein effect [1]. Furthermore, efficient long-range spin coherent applications [2] require spin transport protected from backscattering. A first step in this direction using strong Rashba spin-orbit coupling (SOC) has been made in heavy-group atoms grown epitaxially on group IV surfaces, like $\beta$ - $\mathrm{Pb} / \mathrm{Ge}(111)[3,4]$ or $\mathrm{Au} / \mathrm{Si}(111)$ [5]. Surprisingly, in less dense $1 / 3$ monolayer (ML) phases, effects produced by SOC were overlooked while reversible phase transitions with temperature were found [6-9], accompanied by metal-insulator transitions [10-12] and possible magnetic orderings $[13,14]$.

At room temperature, 1/3 ML coverage of $\mathrm{Pb}$ or $\mathrm{Sn}$ grown on top of $\mathrm{Si}(111)$ or $\mathrm{Ge}(111)$ displays an isoelectronic $\sqrt{3} \times \sqrt{3} R 30^{\circ}$ reconstruction. The host atoms occupy the $T_{4}$ sites in an hexagonal array [6], the so-called $\alpha$ phase [see Fig. 1(d)]. The three Si dangling bonds in the top Si layer next to the metal atom are saturated, leaving a free unsaturated electron at each $T_{4}$ site. These systems are thus expected to present a half filled surface band well separated from Si bands. This expectation is in contrast with experimental evidence of an insulating ground state in $\mathrm{Sn}$ on $\mathrm{Ge}(111)$ [10] and $\mathrm{Si}(111)[11,16]$.

This contradictory behavior was attributed to electronic correlations [12,17]. At a low temperature, the situation is usually complicated by the presence of a reversible structural transition from $\sqrt{3} \times \sqrt{3}$ to $3 \times 3$ periodicity $[6,7,18,19]$. Its origin is still debated and could be either due to the freezing of an out-of-plane phonon mode [9] or produced by long-range electronic correlations $[17,20]$, both favoring a charge-density wave having the so-called one-up-two-down atom configuration. Surprisingly, despite the presence of heavy atoms and substrate-induced broken inversion symmetry, only one theoretical work (restricted to the $\sqrt{3} \times \sqrt{3}$ reconstruction) treats $a b$ initio and on equal footing relativistic and correlation effects as well as the interaction with the substrate [21]. Finally, despite many theoretical calculations for $1 / 3 \mathrm{ML}$ $\mathrm{Pb} / \mathrm{Si}(111)[9,17,21]$, the small size of the $3 \times 3$ domains has prevented, up to now, the experimental spectroscopy of their electronic structure.

Here we study the $1 / 3 \mathrm{ML} \alpha-\mathrm{Pb} / \mathrm{Si}(111)$ by scanning tunneling microscopy and spectroscopy (STM and STS) and the fully relativistic density functional theory (DFT). Experimental results down to $300 \mathrm{mK}$ reveal the $3 \times 3$ ground state to be a correlated metal not undergoing a Mott transition. It reveals a largely depressed density of states (DOS) at the Fermi level $E_{F}$, where well-defined quasiparticles exist, forming two large Fermi surfaces with dominant in-plane spin polarization and opposite helicities.

The preparation of the $\alpha-\mathrm{Pb} / \mathrm{Si}(111)$ surface is described in Supplemental Material [22]. It is known that large $\sqrt{3} \times$ $\sqrt{3}$ areas with a low defect density cannot be grown $[18,19]$ in contrast to $\mathrm{Pb}$ or $\mathrm{Sn} / \mathrm{Ge}(111)$, or $\mathrm{Sn} / \mathrm{Si}(111)$. Below $86 \mathrm{~K}$, the $\sqrt{3} \times \sqrt{3}$ regions transit to a $3 \times 3$ structure $[18,19]$ [see Fig. 1(d)]. The measurements were performed at $0.3,2$, and $4 \mathrm{~K}$ with metallic PtIr or $\mathrm{W}$ tips. The $d I / d V(V)$ spectra shown hereafter were obtained by a numerical 


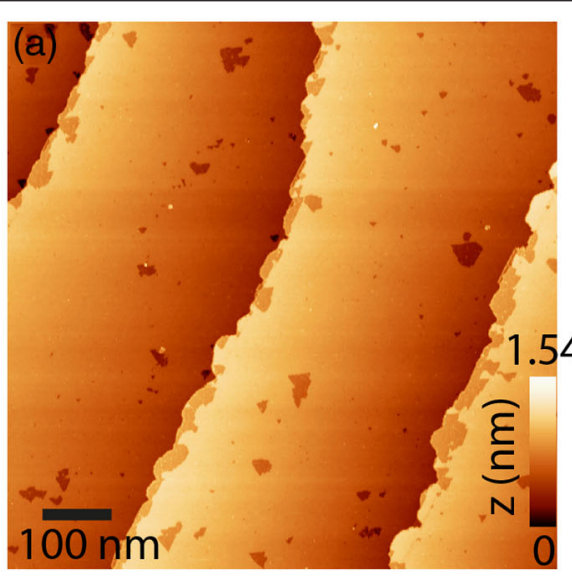

(d)
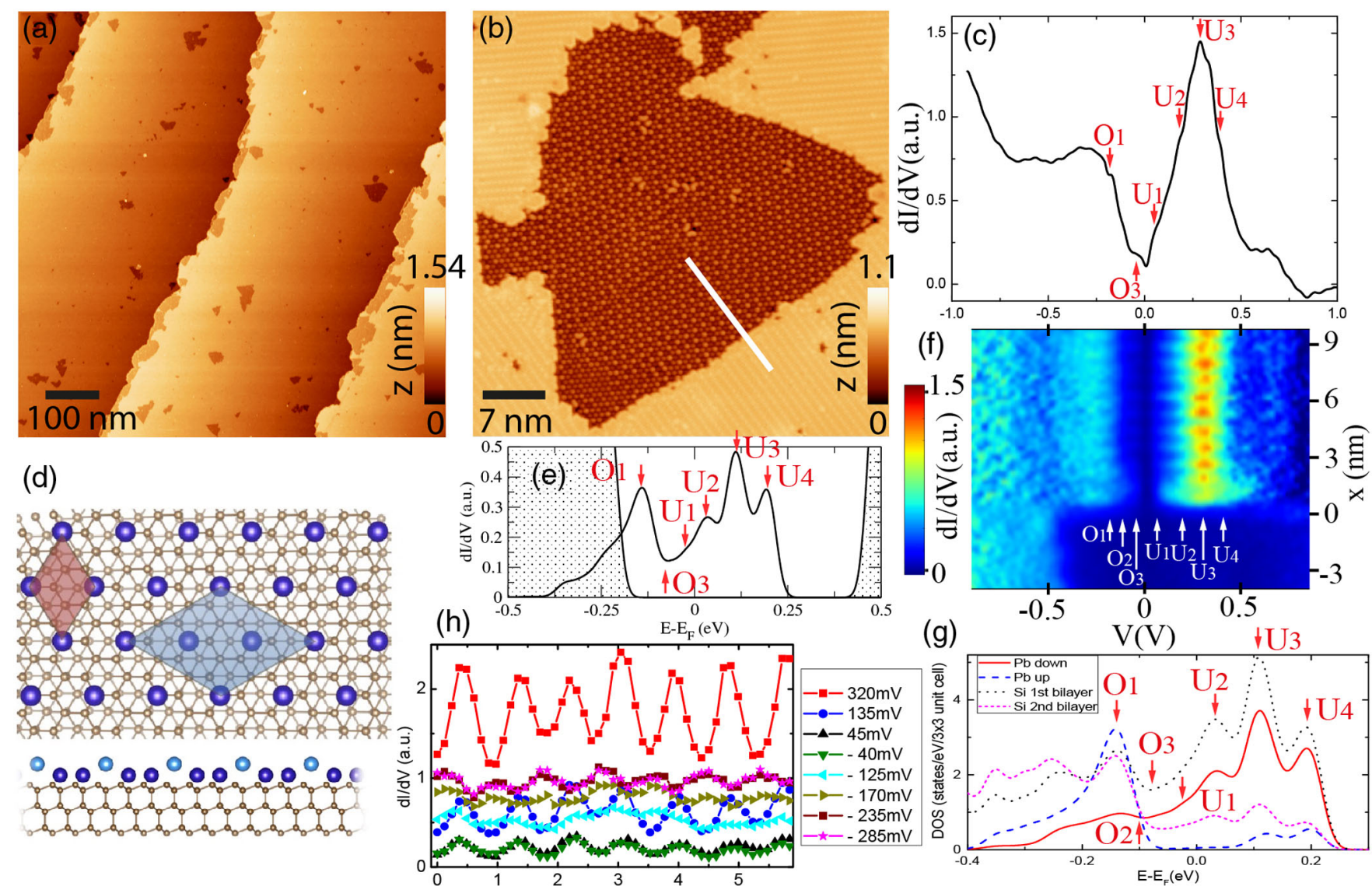

FIG. 1. Measurements at $T=4.2 \mathrm{~K}$. (a) $900 \times 900 \mathrm{~nm}^{2} \mathrm{STM}$ topographic map of the sample (scanning $V=-1.0 \mathrm{~V}, I=20 \mathrm{pA}$ ). (b) $48 \times 48 \mathrm{~nm}^{2}$ topography of a $3 \times 3$ area showing $\mathrm{Pb}$ up and down atoms. (c) STS $d I / d V(V)$ conductance spectra between $[-0.9,+0.9] \mathrm{V}$ inside a $3 \times 3$ area, spatially averaged over $9 \mathrm{~nm}^{2}$ (set point $V=-1.0 \mathrm{~V}, I=200 \mathrm{pA}$ ). (d) Top: Top view of the hightemperature $\sqrt{3} \times \sqrt{3} R 30^{\circ}$ (red) and low-temperature $3 \times 3$ (blue) unit cells of $1 / 3 \mathrm{ML} \mathrm{Pb} / \mathrm{Si}(111)$. Bottom: Side view of the $3 \times 3$. Small (large) circles represent $\mathrm{Si}(\mathrm{Pb})$ atoms. (e) Computed STS spectrum for the $3 \times 3 \alpha$ phase using Tersoff-Hamann (TH) theory [15] in the DFT $+U$ approximation, including SOC effects for a supercell having three Si double layers. The gray region is the TH calculation for a semi-infinite $\operatorname{Si}(111)$ slab. (f) Color plot representing the conductance of $62 d I / d V(V)$ spectra measured along the $13.8 \mathrm{~nm}$ white line shown in (b), across the interface between a $3 \times 3$ and a $\sqrt{7} \times \sqrt{3}$ domain. The right axis indicates the distance $x$ from the boundary (being at $x=0 \mathrm{~nm}$ ) at which each spectrum is measured. The voltage axis is identical to the one used in (c), enabling a direct comparison of all spectral features. Each spectrum is the average of 15 spectra measured over a width of $5 \mathrm{~nm}$ perpendicular to the white line. The $3 \times 3$ band structure establishes sharply over less than $1 \mathrm{~nm}$ from the interface. Periodic oscillations appear along $x$ between $[-0.3,+0.4] \mathrm{V}$ reflecting the different LDOS at the $\mathrm{Pb}$ up and down atoms, seen in $(\mathrm{g})$ presenting the calculated LDOS projected on atomic sites. (h) presents cuts at various energies in map (f) enabling one to better see the oscillations in (f).

derivation of the raw $I(V)$ curves. Negative (positive) bias voltage corresponds to occupied (empty) sample states.

A $900 \times 900 \mathrm{~nm}^{2}$ STM topography of the studied samples is shown in Fig. 1(a). The $3 \times 3$ areas are surrounded by metallic $\sqrt{7} \times \sqrt{3}$ domains enabling efficient charge evacuation [30]. $3 \times 3$ regions of size $25-100 \mathrm{~nm}$, such as the main one seen in Fig. 1(b), were chosen for the spectroscopic studies. This size range is similar to the one presented in Ref. [18]. Intrinsic $3 \times 3$ spectroscopic features were measured far enough from boundaries with neighboring $\sqrt{7} \times \sqrt{3}$ domains.

A characteristic $d I / d V$ spectrum measured locally in clean $3 \times 3$ areas is presented in Fig. 1(c). It reveals a strongly depressed DOS at $E_{F}$ not reaching zero, demonstrating the correlated semimetallic character of the surface. The most prominent feature is a peak at $+290 \mathrm{meV}$ labeled $U_{3}$. Kinks were always observed at -170 and $-60 \mathrm{meV}$ labeled $O_{1}$ and $\mathrm{O}_{3}$, respectively. At a larger binding energy $(-420<\mathrm{V}<$ $-250 \mathrm{meV}$ ), the conductance increases, forming a broad peak with internal fine features. The DOS then remains flat with small oscillations until $V \simeq-660 \mathrm{meV}$ and increases strongly for $V<-660 \mathrm{meV}$ due to $\mathrm{Si}$ bands [31]. In the unoccupied states, several reproducible small kinks are observed around the peak $U 3$ at $+55,+180$, and $+400 \mathrm{meV}$ denoted, respectively, $U 1, U 2$, and $U 4$. For $V>400 \mathrm{meV}$, a continuous drop in conductance occurs until about $550 \mathrm{meV}$. The conductance remains low but nonzero until $650 \mathrm{meV}$ and then drops, leading to negative $d I / d V$ above $V \simeq 750 \mathrm{meV}$ and 
below $1 \mathrm{~V}$. Above $1 \mathrm{~V}$, a strong increase signals Si bands (see Sec. II in Supplemental Material for additional data and a discussion of the small features [22]).

In principle, the absolute energies of these features must be taken with caution, as the electric field of the STM tip can induce a band bending at the surfaces of gapped solids like semiconductors [31] or Mott systems [32]. However, here $3 \times 3$ areas cover less than $10 \%$ of the surface, the remaining being the metallic $\sqrt{7} \times \sqrt{3}$ phase becoming superconducting below $1.5 \mathrm{~K}[30,33]$. Furthermore, since $3 \times 3$ areas are semimetallic and not insulating, a small band bending effect is expected. The comparison between STS, ARPES, and DFT $+U$ in $1 / 3 \mathrm{ML}$ Sn/Si(111) shows negligible band bending for the occupied states and a small effect for the unoccupied states [11]. The most important contributions that we found to influence the LDOS of $3 \times 3$ areas are (i) local or extended defects and (ii) dynamical motion of Pb atoms below the tip (see Sec. II in Ref. [22]).

To rationalize the experimental findings, we performed theoretical calculations using QUANTUM ESPRESSO [34]. We included relativistic effects, noncollinear magnetism in the DFT $+U$ approximation, and the $\mathrm{Si}(111)$ substrate (see Supplemental Material [22] and Ref. [9] for more details). We first consider $1 / 3 \mathrm{ML} \mathrm{Pb}$ on $\mathrm{Si}(111)$ in the $\sqrt{3} \times \sqrt{3} R 30^{\circ}$ structure within the spin polarized generalized gradient approximation (GGA). While the structural properties are not affected by relativistic effects (see Supplemental Material [22] and Ref. [9]), SOC has a strong impact on the electronic structure inducing a band splitting as large as $1 / 4$ of the bandwidth [see Fig. 2(a)]. The electron-electron interaction treated in the DFT $+U$ approximation or using the HSE06 has minor effects on the $\sqrt{3} \times \sqrt{3}$ surface-band electronic structure [22].

To understand the microscopic mechanisms behind the $3 \times 3$ reconstruction, we first neglect SOC and study the lattice deformation within the GGA and local-density approximation (LDA) approximations. Using the GGA, we find a stabilization of the $3 \times 3$ reconstruction (energy gain $5 \mathrm{meV}$ per $\mathrm{Pb}$ atom, difference in height of $\mathrm{Pb}$ atoms $\Delta h \sim 0.24 \AA$ ) in agreement with previous results [9]. Despite the indistinguishable electronic structure, the LDA does not lead to a structural transition, ruling out Fermi surface effects. Although the structural $3 \times 3$ distortion is well reproduced in paramagnetic GGA neglecting SOC, the resulting surface DOS is clearly metallic with no depressed DOS at $E_{F}$ (see Supplemental Material [22]), in qualitative disagreement with the experiment. Including SOC splits the topmost peak in the empty DOS by $\approx 0.09 \mathrm{eV}$, generating a three-peak structure but keeping a metallic state [see Fig. 2(b)]. Within the GGA, we also considered possible magnetic instabilities. Even if we could stabilize different magnetic solutions, those were always higher in energy than the paramagnetic one [22]. We then consider the relativistic rotational-invariant formulation of the GGA $+U$ approximation [35]. We evaluated the $U$ parameter self-consistently and obtained $U \sim 1.75 \mathrm{eV}$.
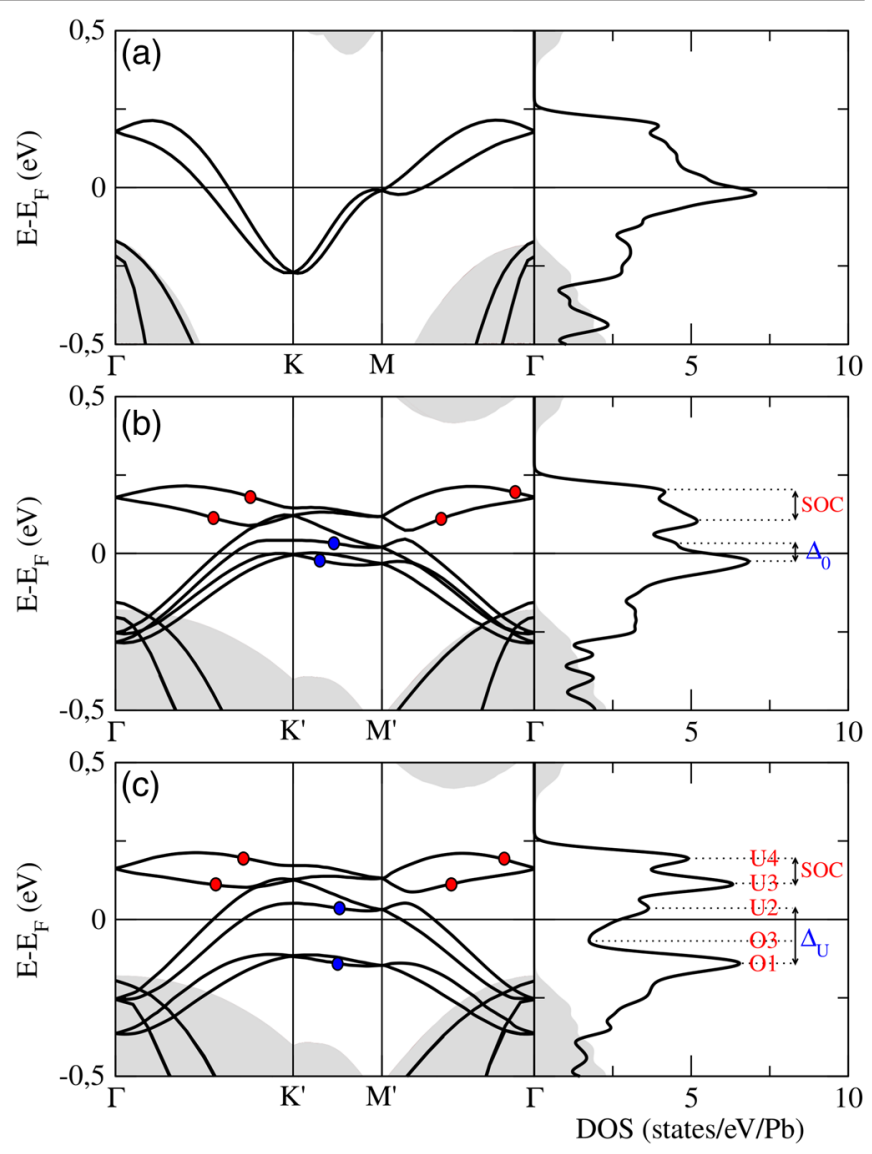

FIG. 2. Fully relativistic electronic structure for the $\sqrt{3} \times$ $\sqrt{3} R\left(30^{\circ}\right)$ (a) and $3 \times 3$ (b) phases with $U=0 \mathrm{eV}$. In (c) $3 \times$ 3 with $U=1.75 \mathrm{eV}$. Dots in (b) and (c) label the k points leading to peaks in the DOS (right panels). The projected Si bulk band structure (and DOS) is indicated by gray areas.

We optimized the internal parameters and again found that the paramagnetic $3 \times 3$ phase is the ground state $(8 \mathrm{meV} / \mathrm{Pb}$ energy gain and $\Delta h \sim 0.32 \AA$ ). Since the energy gain is larger at $U \sim 1.75 \mathrm{eV}$ than at $U=0 \mathrm{eV}$, the local Coulomb repulsion enhances the distortion. Figure 2(c) shows the electronic structure. The Hubbard repulsion lowers the energies of half of the occupied bands, reducing the DOS near $E_{F}$. It is possible to probe directly the SOC $\left(\Delta_{\mathrm{SOC}}\right)$ and Hubbard $\left(\Delta_{U}\right)$ splittings at selected $\mathbf{k}$ points by looking at the corresponding features induced in the empty and occupied DOS. As presented in the right panel in Fig. 2(c), the main experimental features of the $\mathrm{Pb}-\alpha$ phase DOS are reproduced by the calculations: one peak $O_{1}$ in the occupied states, a depressed DOS $O_{3}$ around $E_{F}$, and a three-peak structure $U_{2}$, $U_{3}, U_{4}$ centered around the prominent peak $U_{3}$ in the empty states. We underline that the spin-orbit splitting is about $50 \%$ of the Hubbard one, demonstrating the crucial importance of including both effects in the band structure calculation.

To compare in more detail the theory and experiment, the Tersoff-Hamann (TH) simulation [15] of the STS spectra is presented in Fig. 1(e). A good qualitative agreement exists for the energies at which spectral features are observed in 

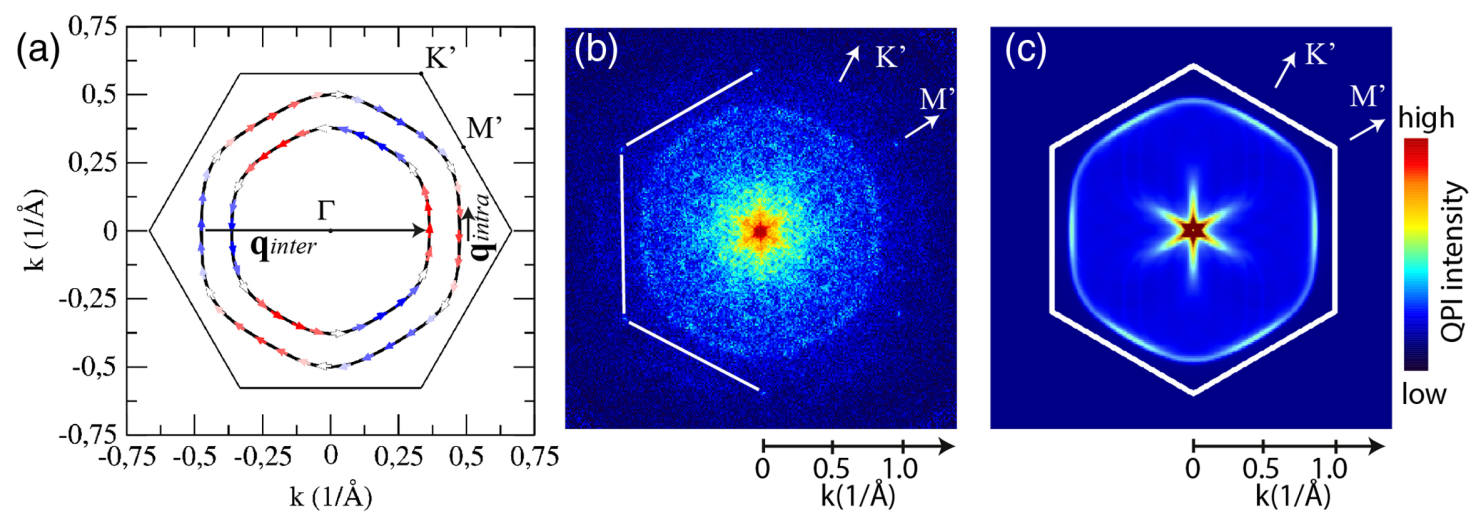

FIG. 3. (a) $\mathrm{DFT}+U$ Fermi surface of $\mathrm{Pb}-3 \times 3 / \mathrm{Si}(111)$ including spins polarization (arrows). The out-of-plane spin component is at most $1 \%$ of the in-plane one. White arrows, $100 \%$ in-plane polarization; blue and red arrows, opposite out-of-plane components. Black arrows $\mathbf{q}_{\text {inter }}$ and $\mathbf{q}_{\text {intra }}$, scattering vectors corresponding to scalar impurities. (b) Symmetrized Fourier transform of a $60 \times 60 \mathrm{~nm}^{2}$ $d I / d V(V=0)$ map measured by STS at $T=0.3 \mathrm{~K}$ and $B=0.5 \mathrm{~T}$, corresponding to quasiparticle interference at $E=E_{F}$. (c) Calculated quasiparticle interference map at $E=E_{F}$ assuming pure scalar impurity scattering.

the occupied states. The agreement concerning their amplitude and shape is less good, since $O_{1}$ is a kink in the experiment. Most likely, this is because the $\mathrm{TH}$ approximation is not valid anymore close to $O_{1}$ : Figure 2(c) shows that states close to $O_{1}$ have a large in-plane momentum $\left\|\mathbf{k}_{\|}\right\| \simeq \Gamma M^{\prime}$. Thus, tip states with $l \neq 0$ angular momentum can significantly contribute to $d I / d V(V)$, invalidating the TH assumptions [36]. Below $O_{1}$ in STS, a broad peak with small oscillating features ends up around $-0.43 \mathrm{eV}$ in agreement with the calculated halfbandwidth. The small oscillations come from surface $\mathrm{Si}$ atoms [see Fig. 1(g)] and continue for $-0.70<V<$ $-0.43 \mathrm{eV}$ (see Fig. 8 in Ref. [22]). The LDOS increases further for $V<-0.70 \mathrm{eV}$ due to bulk Si bands. For the unoccupied states, the main shape of a large peak $U_{3}$ with three additional features $U_{1}, U_{2}$, and $U_{4}$ agrees with the calculations, although finite lifetime effects most likely broaden the real levels (see Sec. VIII and Fig. 10 in Ref. [22]). Energies $U_{i}$ are found larger in STS due to small band bending, as observed in Refs. [10,11]. The measured upper half-bandwidth is $0.50 \mathrm{eV}$, while the calculated one is $0.35 \mathrm{eV}$. One cannot exclude that DFT $+U$ slightly underestimates the bandwidth, because HSE06 calculations show a larger bandwidth mostly for empty states (see [22]).

We now address the conductance oscillations measured between the $\mathrm{Pb}$ up and down atoms shown in Fig. 1(f), which strongly support our interpretation of the LDOS structure. For electron energies $O_{2}<E<U_{4}$, DFT $+U$ predicts a larger LDOS on the two $\mathrm{Pb}$ down atoms than on the $\mathrm{Pb}$ up ones [Fig. 1(g)]. The opposite occurs for $E<\mathrm{O}_{2}$. The local measurements presented in Fig. 1(f) and energy cuts in Fig. 1(h) agree well with these predictions in the range $[-0.3 ;+0.4] \mathrm{eV}$. Finally, the experimental $V$-shaped DOS around $E_{F}$ on the meV scale is typical of correlated metals [37] and can be modeled using a dynamical Coulomb blockade [38].
The $\mathrm{Pb}-\alpha$ phase being a correlated 2D metal, one may wonder whether or not it exhibits well-defined quasiparticle excitations at $E_{F}$. We answered this question by measuring quasiparticle interference (QPI) $d I / d V(V=0)$ map by STS and performing its Fourier transform (FT) [39]. Here we expect nonmagnetic scalar scattering to be the most dominant scattering process. We verified that magnetic scatterers and spin-orbit scatterers do not contribute significantly to the FT-QPI maps using a similar approach as in Ref. [40].

The calculated Fermi surface of the $3 \times 3$ reconstruction is shown in Fig. 3(a). It consists of two hexagons, carrying essentially in-plane spin polarization with opposite helicities: It is thus almost of pure Rashba type. We then calculated the joint DOS due to scattering by scalar impurities, namely, $\chi(\mathbf{q})=\left(\mathbf{1} / \mathbf{N}_{\mathbf{K}}\right) \sum_{\mathbf{k n}, \mathbf{k}^{\prime} \mathbf{m}}\left|\mathbf{M}_{\mathbf{k n}, \mathbf{k}^{\prime} \mathbf{m}}\right|^{\mathbf{2}} \delta\left(\epsilon_{\mathbf{k n}}-\mathbf{E}_{\mathbf{F}}\right) \delta\left(\epsilon_{\mathbf{k}^{\prime} \mathbf{m}}-\mathbf{E}_{\mathbf{F}}\right)$, where $\mathbf{q}=\mathbf{k}-\mathbf{k}^{\prime}, \epsilon_{\mathbf{k} n}$ are the relativistic DFT $+U$ electronic bands, and $M_{\mathbf{k} n, \mathbf{k}^{\prime} m}=\left\langle\mathbf{k} n\left|\sigma_{0} \exp \left\{i\left(\mathbf{k}^{\prime}-\mathbf{k}\right) \cdot \mathbf{r}\right\}\right| \mathbf{k}^{\prime} m\right\rangle$ with $\sigma_{0}$ the identity matrix in spin space. In the pure Rashba case, one has $M_{\mathbf{k} n, \mathbf{k}^{\prime} m}=1+(-1)^{n-m} \cos \left[\phi(\mathbf{k})-\phi\left(\mathbf{k}^{\prime}\right)\right]$ and $\phi(\mathbf{k})=\left(k_{x}+i k_{y}\right) / k$. In Fig. 3(c), we display $\chi(\mathbf{q})$ as a color plot. The hexagonal feature corresponds to interband $(m \neq n)$ scattering between segments of different hexagons of the Fermi surface having parallel spins [see $\mathbf{q}_{\text {inter }}$ in Fig. 3(a)]. Near $\mathbf{q}=\mathbf{0}$, a star-shaped feature occurs due to intra Fermi sheet scattering along the straight side of each hexagon [see $\mathbf{q}_{\text {intra }}$ in Fig. 3(a)].

The threefold symmetrized experimental FT-QPI map is shown in Fig. 3(b) (for raw data and details, see Sec. IV in Ref. [22]). A hexagon of maximal radius $\simeq 0.8 \AA^{-1}$ is found, in good agreement with Fig. 3(c). Near $\mathbf{q}=\mathbf{0}$, a starshaped feature is seen. The remaining weaker scattering signal probably comes from extended defects. We checked that it cannot be produced assuming unpolarized spin bands (see Sec. IV [22-29]). The good agreement between calculated and experimental FT-QPI maps demonstrates 
(i) well-defined quasiparticles exist at $E_{F}$ forming two large hexagonal Fermi sheets with opposite in-plane spin-helicities and (ii) mainly scalar scatterers.

In conclusion, using STS experiments and DFT calculations, we showed that the $\mathrm{Pb}$-substrate interaction, noncollinear spin-orbit coupling, and correlation effects are all mandatory to describe the electronic structure of the $1 / 3$ $\mathrm{ML} \mathrm{Pb} / \mathrm{Si}(111)$ surface and its reconstructions. The lowtemperature ground state of the $3 \times 3$ reconstruction is a strongly correlated metal with well-developed lower and upper Hubbard bands coexisting with a charge-density wave. By comparing calculated quasiparticle interference with the Fourier transform of STS data, we demonstrated the occurrence of two large hexagonal Fermi surfaces with in-plane spin polarization and opposite helicities.

We acknowledge CINECA (ISCRA initiative), CINES, IDRIS, TGCC, and PRACE (Proposal 2017174186) for computing resources. This work was supported by French state funds managed by the ANR within the Investissements d'Avenir program under reference ANR-11-IDEX-0004-02 and, more specifically, within the framework of the Cluster of Excellence MATISSE led by Sorbonne université, RODESIS ANR-16-CE30-0011-01, and MISTRAL. We acknowledge M. Tringides, S. Pons, A. Tejeda, M. Cococcioni, and A. Smogunov for useful discussions.

Recently, we became aware of a work performed on a similar system to ours: $1 / 3 \mathrm{ML} \mathrm{Sn} / \mathrm{Si}(111)$ reaching a Mott insulator state for heavily $n$-doped substrate [42]. Following old [12] and new theoretical predictions [43] a very exciting perspective to our work is to study whether unconventional superconductivity could be induced or not in triangular lattice systems such as the $1 / 3 \mathrm{~Pb}$ or $\mathrm{Sn}$ grown on $\mathrm{Si}(111)$ or $\mathrm{Ge}(111)$.

*christophe.brun@upmc.fr

†matteo.calandra@insp.upmc.fr

[1] A. Soumyanarayanan, N. Reyren, A. Fert, and C. Panagopoulos, Nature (London) 539, 509 (2016).

[2] S. A. Wolf et al., Science 294, 1488 (2001).

[3] K. Yaji, Y. Ohtsubo, S. Hatta, H. Okuyama, K. Miyamoto, T. Okuda, A. Kimura, H. Namatame, M. Taniguchi, and T. Aruga, Nat. Commun. 1, 1 (2010).

[4] X.-Y. Ren, H.-J. Kim, S. Yi, Y. Jia, and J.-H. Cho, Phys. Rev. B 94, 075436 (2016).

[5] L. V. Bondarenko et al., Sci. Rep. 3, 1826 (2013).

[6] J. M. Carpinelli, H. H. Weitering, E. W. Plummer, and R. Stumpf, Nature (London) 381, 398 (1996).

[7] J. M. Carpinelli, H. H. Weitering, M. Bartkowiak, R. Stumpf, and E. W. Plummer, Phys. Rev. Lett. 79, 2859 (1997).

[8] G. Profeta, L. Ottaviano, and A. Continenza, Phys. Rev. B 69, 241307 (2004).

[9] P. Cudazzo, G. Profeta, and A. Continenza, Surf. Sci. 602, 747 (2008).
[10] R. Cortés, A. Tejeda, J. Lobo, C. Didiot, B. Kierren, D. Malterre, E. G. Michel, and A. Mascaraque, Phys. Rev. Lett. 96, 126103 (2006).

[11] S. Modesti, L. Petaccia, G. Ceballos, I. Vobornik, G. Panaccione, G. Rossi, L. Ottaviano, R. Larciprete, S. Lizzit, and A. Goldoni, Phys. Rev. Lett. 98, 126401 (2007).

[12] G. Profeta and E. Tosatti, Phys. Rev. Lett. 98, 086401 (2007).

[13] G. Li, P. Höpfner, J. Schäfer, C. Blumenstein, S. Meyer, A. Bostwick, E. Rotenberg, R. Claessen, and W. Hanke, Nat. Commun. 4, 1620 (2013).

[14] J.-H. Lee, H.-J. Kim, and J.-H. Cho, Phys. Rev. Lett. 111, 106403 (2013).

[15] J. Tersoff and D. R. Hamann, Phys. Rev. B 31, 805 (1985).

[16] A. B. Odobescu, A. A. Maizlakh, N. I. Fedotov, and S. V. Zaitsev-Zotov, Phys. Rev. B 95, 195151 (2017).

[17] P. Hansmann, T. Ayral, L. Vaugier, P. Werner, and S. Biermann, Phys. Rev. Lett. 110, 166401 (2013).

[18] I. Brihuega, O. Custance, R. Pérez, and J. M. GómezRodrígues, Phys. Rev. Lett. 94, 046101 (2005).

[19] I. Brihuega, O. Custance, M. Ugeda, and J. GómezRodrígues, Phys. Rev. B 75, 155411 (2007).

[20] P. Hansmann, T. Ayral, L. Vaugier, P. Werner, and S. Biermann, J. Phys. Condens. Matter 25, 094005 (2013).

[21] D. I. Badrtdinov, S. A. Nikolaev, M. I. Katsnelson, and V. V. Mazurenko, Phys. Rev. B 94, 224418 (2016).

[22] See Supplemental Material at http://link.aps.org/ supplemental/10.1103/PhysRevLett.120.196402 for various additional details regarding experimental results and theoretical aspects. The supplemental file includes Refs. [23-29] not referred to in the main text.

[23] O. Custance, J. M. Gómez Rodríguez, A. M. Baróa, L. Juré, P. Mallet, and J.-Y. Veuillen, Surf. Sci. 482, 1399 (2001).

[24] R. Wiesendanger, Scanning Probe Microscopy and Spectroscopy: Methods and Applications (Cambridge University Press, Cambridge, England, 1994).

[25] Y. Kohsaka et al., Nature (London) 454, 1072 (2008).

[26] H. J. Monkhorst and J. D. Pack, Phys. Rev. B 13, 5188 (1976).

[27] L. Lin, J. Chem. Theory Comput. 12, 2242 (2016).

[28] D. R. Hamann, Phys. Rev. B 88, 085117 (2013).

[29] M. Schlipf and F. Gygi, Comput. Phys. Commun. 196, 36 (2015).

[30] C. Brun, T. Cren, and D. Roditchev, Supercond. Sci. Technol. 30, 013003 (2017).

[31] S. Modesti, H. Gutzmann, J. Wiebe, and R. Wiesendanger, Phys. Rev. B 80, 125326 (2009).

[32] I. Battisti, V. Fedoseev, K. M. Bastiaans, A. de la Torre, R. S. Perry, F. Baumberger, and M. P. Allan, Phys. Rev. B 95, 235141 (2017).

[33] C. Brun et al., Nat. Phys. 10, 444 (2014).

[34] P. Giannozzi et al., J. Phys. Condens. Matter 21, 395502 (2009).

[35] M. Cococcioni and S. de Gironcoli, Phys. Rev. B 71, 035105 (2005).

[36] C. Chen, Introduction to Scanning Tunneling Microscopy, Oxford Monographs on the Physics and Chemistry of Materials, 2nd ed. (Oxford University, New York, 2008), pp. 153-167.

[37] P. A. Lee and T. Ramakrishnan, Rev. Mod. Phys. 57, 287 (1985). 
[38] C. Brun, K. H. Muller, I. Po Hong, F. Patthey, C. Flindt, and W. D. Schneider, Phys. Rev. Lett. 108, 126802 (2012).

[39] L. Simon, C. Bena, F. Vonau, M. Cranney, and D. Aubel, J. Phys. D 44, 464010 (2011).
[40] Y. Kohsaka, T. Machida, K. Iwaya, M. Kanou, T. Hanaguri, and T. Sasagawa, Phys. Rev. B 95, 115307 (2017).

[41] C. Brand et al., Phys. Rev. B 96, 035432 (2017).

[42] F. Ming et al., Phys. Rev. Lett. 119, 266802 (2017).

[43] X. Cao et al., Phys. Rev. B 97, 155145 (2018). 